\title{
Overcoming Automaticity Through Meditation
}

\author{
Thomas Maran $^{1,2}$ (D) $\cdot$ Martin Woznica ${ }^{3} \cdot$ Sebastian Moder $^{4}\left(\mathbb{C}^{\circ} \cdot\right.$ Marco Furtner $^{4} \cdot$ Elias Jehle $^{5} \cdot$ Stanislaw Hörner $^{6}$. \\ Gregor Hugger $^{3}$
}

Accepted: 4 September 2021 / Published online: 23 September 2021

(c) The Author(s) 2021

\begin{abstract}
Objectives Meditation practice has recently moved into applied research to improve cognitive functions. However, it is a multifaceted practice, with focused attention meditation relying on a sharp focus, and open monitoring meditation relying on a diffuse awareness. This study aims to assess the effects of differential alterations of cognition following distinct meditative training and focuses on practitioners' tendency to fall victim to erroneous automaticity in responding when faced with cognitive conflict.

Methods Seventy-three individuals were randomly assigned to one of three intervention groups (internally focused attention meditation, externally focused attention meditation, open monitoring meditation) or a wait list control group. The meditation groups were trained over the course of 4 weeks and eight sessions. Changes in proneness to erroneous automatic responding were tested using two cognitive performance tasks that induce learned or instructed automaticity (Dot Pattern Expectancy paradigm, NEXT-paradigm).

Results Overall, meditation training generally improved overcoming learned automaticity $(r s=.26-.36, p s=.002-.031)$ but not instructed automaticity compared to the control condition. Furthermore, data suggest open monitoring outperformed focused attention in overcoming learned automaticity in one task $(r \mathrm{~s}=.31-.56, p \mathrm{~s} \leq .001-.009)$.

Conclusions Our results provide evidence for meditative training to facilitate practitioners' ability to select the most appropriate course of action against overlearned habits in light of the peculiarities of their current situation. Open monitoring meditation is a particularly promising avenue for reducing one's liability to erroneous habits.
\end{abstract}

Keywords Cognitive training $\cdot$ Mindfulness $\cdot$ Meditation $\cdot$ Automaticity $\cdot$ Cognitive control

Tremendous effort is needed to change deeply ingrained habitual behaviors. Particularly in the rush of everyday operations and short deadlines, individuals may be tempted to revert to old paradigms and postpone a shift to new and

Thomas Maran

thomas.k.maran@gmail.com

1 Department of Strategic Management and Leadership, University of Innsbruck, Innsbruck, Austria

2 LeadershipWerk, 9490 Vaduz, Liechtenstein

3 Department of Psychology, University of Innsbruck, Innsbruck, Austria

4 Institute of Entrepreneurship, University of Liechtenstein, Vaduz, Liechtenstein

5 Center for Organizational Viability, School of Management and Law, Zurich University of Applied Sciences, Winterthur, Switzerland

6 Department of Psychology, University of Graz, Graz, Austria improved ones to tomorrow again and again. Being able to overcome one's automatic behaviors and to apply new rules in their place is a critical skill. Recently, the processes underlying automatic responses have received increased attention in cognitive psychology (Moors, 2016). Having a prepared behavioral pattern at one's disposal that gets executed in an automatic, reflex-like manner enables quick and efficient responses (Meiran et al., 2015). These response patterns indicate a high-level preparedness to perform a desired action (Logan, 1978), and they can form in a twofold manner. Learned automaticity is created through repeated execution of a behavior in response to a trigger (Servan-Schreiber et al., 1996). With increased repetition, the cognitive effort required to decide on a behavior or movement and to implement it diminishes, transforming the response over time from a deliberate action into an overlearned habit (Packard \& Goodman, 2013) represented in long-term memory (Meiran et al., 2015). More recently, another possible option to prepare an automated response has been identified: instructed 
automaticity (Meiran et al., 2017). This type of automaticity stems from the intention to act being held in working memory (Meiran et al., 2012). By keeping the instructions on how to deal with an arising task or challenge in mind, one can execute the appropriate behavior rapidly without having to have learned it through repeated execution. Many actions in everyday life are characterized by routines and repeating processes; they reflect automated reflex-like behaviors. Running on autopilot is unavoidable and usually beneficial: deliberate action requires more mental effort, which is inherently aversive (Inzlicht et al., 2015; Kurzban, 2016). However, the complexity of daily life can present individuals with unexpected challenges. When situations change, new opportunities or demands may prompt an adaptation of one's strategy. Some situations trigger automated responses that cause individuals to miss better opportunities or even cause harm. Highly automated behaviors, once triggered, prevent individuals from adapting their reaction when a different response would be required (Meiran et al., 2012). Although attempts at using cognitive training to overcome reflexive responses are numerous, their transferability to general cognition and performance in natural situations is weak (MelbyLervåg et al., 2016; Sala \& Gobet, 2019). Meditation may be a more promising candidate; different meditation techniques can impact cognition in different ways (Hommel \& Colzato, 2017). However, existing studies in this field often bear certain limitations in their approaches. Some lack a clear delineation of different techniques (e.g., Ainsworth et al., 2013), while others examine the effects of only a single short session of meditation (e.g., Colzato et al., 2012).

Meditation is a way to cultivate mindfulness and originates in the Buddhist tradition. Mindfulness was translated from the Pāli term sati, which translates to "attentiveness," "awareness," "memory," "calling to mind," or "keeping in mind" (Anālayo, 2003; Gethin, 2011). From a cognitive perspective, meditation involves the control of attention and awareness (e.g., Cahn \& Polich, 2009; Holas \& Jankowski, 2013; Isbel \& Mahar, 2015) to remain centered on the present moment by mentally responding to arising stimuli in a specific way (Tang et al., 2007). Today, several models attempt to capture the cognitive mechanisms behind mindful meditation (e.g., Bishop et al., 2004; Grabovac et al., 2011; Holas \& Jankowski, 2013; Isbel \& Mahar, 2015; Kang et al. 2013; Shapiro et al., 2006), though the lack of a generally accepted definition of mindfulness hampers these attempts. For example, some of them emphasize attention-regulation processes (e.g., Kang et al., 2013), and others focus more on metacognition (e.g., Holas \& Jankowski, 2013).

Being mindful requires the practitioner to keep their attention on the target of observation and, when the mind drifts away, to gently bring their attention back to the original target (Kang et al., 2013). Metacognition may play a key role in coordinating attentional processes and executive functions to sustain a de-centered observation in mindfulness meditation (Holas \& Jankowski, 2013; Lutz et al., 2015). In other words, meditation trains one to orchestrate the cognitive operations in order to achieve the intended state of mindfulness. Recent results strongly support that meditation affects the performance of cognitive operations and shapes the way individuals deal with cognitive conflicts (Chiesa et al., 2011). For example, meditation training has been shown to alter attentional performance (e.g., Sumantry \& Stewart, 2021) and executive functions (e.g., Gallant, 2016). However, there are also contrary findings (e.g., Gallant, 2016); this indicates that more sophisticated investigations are required to uncover subtle aspects of attention control resulting from distinct meditative practices that might occur when attention gets challenged by automaticity.

Buddhist scholars recognize two general styles of meditation: samatha, or mental concentration; and vipassana, or insight meditation (Anālayo, 2003). It is said that samatha leads to mental calmness, whereas vipassanā is regarded as a tool to understand the true nature of one's mind (Nyanaponika, 1962). Their secular counterparts are called focused attention (FA) and open monitoring (OM), respectively (Lutz et al., 2008). During FA meditation, one's attention is constantly fixated on an object, either on an internal one (e.g., one's breath) or on an external one (e.g., a candle's flame). In contrast, there is no distinct object to focus on during $\mathrm{OM}$ meditation. Instead, OM practitioners train metacognitive self-awareness: they aim to stay attentive to anything that occurs in their experience from moment to moment. It involves noticing the occurrence and intensity of arising sensations such as emotional tones or intrusive thoughts. Both techniques ask practitioners to put specific constraints on their mental flow. While meditating, occasional thoughts will inevitably capture the practitioner's attention (Christoff et al., 2016). During FA meditation, the arising of a thought or other distraction indicates that the meditator has lost control of their attention and should re-focus their attention on the intended object. Thus, the instructions in FA meditation mainly consist of focusing and readjusting. In contrast, during OM meditation, all arising impulses should be recognized and observed without interfering with them. Here, a loss of control is indicated by the meditator engaging with the arising sensations and expanding them into a "story." Therefore, before the readjustment, OM meditators have to notice the content of their mental flow, thereby allowing some timely dynamic in it so that the spontaneous thought passes on. The instructions for $\mathrm{OM}$ consist of observing, noticing, and letting go, providing an additional task compared to FA.

Both types of meditation practice should rely on and train different cognitive operations (Fox et al., 2016). When faced with a conflict caused by either learned or instructed automaticity, training a clear awareness of 
arising action tendencies without engaging in their execution might result in less erroneous automatic responding. FA trains concentration toward one salient object exclusively. Inversely, when asked to select an appropriate response, $\mathrm{OM}$ practice should provide the necessary flexibility to restrain the tendency to execute an automated response more strongly than FA practice, since OM emphasizes self-monitoring in a non-judgmental, presentoriented manner.

This randomized controlled intervention study investigates how different techniques of meditation differentially affect individuals' preparedness to overcome reflexive behaviors stemming from both learned and instructed automaticity. We hypothesize that meditation practice regardless of technique improves overcoming automaticity compared to a control condition and that open monitoring practice is more effective for overcoming automaticity than focused attention practice.

\section{Method}

\section{Participants}

To be able to investigate different techniques of meditation separately, the most promising avenue is recruiting meditation-naive subjects and training them in either style (Ainsworth et al., 2013). Participants were recruited at an informational meeting. Prospective participants got their questions answered and were informed about meditation, the procedure, and the support by the instructor. It was emphasized that it was of great importance to complete the entire program (personally and with respect to this investigation) and that they would be excluded if they missed the group session three times. In total, 8 subjects abandoned the study by themselves (OM: 2 females; $\mathrm{FA}_{\mathrm{in}}$ : 2 females, 1 male; FA ${ }_{\text {ex }}: 2$ females, 1 male); none had to be excluded for non-compliance. All participants in the final sample $(N=73)$ were meditation-naive individuals and employed at the time of the experiment. Four groups were formed randomly. Three of them learned a different meditation technique each, and the fourth one served as a control group: (1) open monitoring (OM) with 17 subjects $(M=26.2, S D=7.9$ years $),(2)$ focused attention internal (on breathing; $\mathrm{FA}_{\text {in }}$ ) with 18 subjects $(M=24.3, S D=3.3$ years), (3) focused attention external (on candle's flame; $\mathrm{FA}_{\mathrm{ex}}$ ) with 19 subjects $(M=25.5$, $S D=4.1$ years), and (4) a wait list control group with 19 subjects $(M=24.0, S D=4.0$ years). Sample sizes of comparable intervention studies are in a similar range (e.g., Ainsworth, et al., 2013; Jensen et al., 2012).

\section{Procedure}

In order to ensure a clear separation of FA and OM meditation, an experienced meditation teacher recorded meditation instructions based on the program of S. N. Goenka (Hart, 1987). Each session began and ended with a short phase of lying down. When carried out before sitting, this makes it easier to initiate the self-observation; at the end, it prolongs and deepens the practice. The first two introductory sessions were identical for all groups. From the third session onward, the content of the instructions was separated; however, the instructions for each session were similar in length and structure across groups. All sessions were 30 to $35 \mathrm{~min}$ long, which exceeds those used in comparable investigations (e.g., Ainsworth, et al., 2013; Jensen et al., 2012; Morrison et al., 2014). The participants were asked to meditate independently at home every day using the provided audio files.

In the first two sessions of our intervention $\left(\mathrm{T}_{1}\right.$ and $\left.\mathrm{T}_{2}\right)$, the participants were advised to guide their attention toward physical sensations in their body in order to introduce them to the meditative state. This "scanning" of their bodies was combined with gradual movements to help the meditation novices find their preferred posture. The first session $\left(T_{1}\right)$ was performed entirely while lying down, and the second session $\left(\mathrm{T}_{2}\right)$ involved a period of sitting, either on the floor or a chair, based on personal preference.

From $T_{3}$ to $T_{5}$, specific aspects of observation were introduced successively. For the group practicing $\mathrm{FA}_{\mathrm{in}}$, these were different sensations regarding the breath (e.g., its softness, regularity, or phases) in the abdominal $\left(\mathrm{T}_{3}\right)$ and nasal $\left(\mathrm{T}_{4}\right.$ and $\left.\mathrm{T}_{5}\right)$ regions. From $\mathrm{T}_{6}$ on, the meditators were free to choose where to observe the breath. The group practicing $\mathrm{FA}_{\mathrm{ex}}$ was asked to observe various aspects of the candle's flame (e.g., its constancy, brightness, or size). The group practicing OM was advised to observe the sensations that appeared within their experience without letting them become the starting point of a thought or memory. First $\left(\mathrm{T}_{3}\right)$, practitioners were asked to observe physical stimuli (e.g., their points of contact with the floor) and then $\left(\mathrm{T}_{4}\right)$ to recognize the content and quality of each observed stimulus by mentally naming or labeling it. This labeling supports nonevaluation and non-tracking as well as a clearer awareness of the demands posed by their practice (see Creswell et al., 2007). Subsequently $\left(T_{5}\right)$, the observation was extended to include arising emotions or thought impulses.

The last three sessions $\left(\mathrm{T}_{6}-\mathrm{T}_{8}\right)$ within each group were identical. The instructions were no longer expanded upon; instead, the sessions were designed to repeat and deepen the practice. The phases of the teacher's guidance were greatly reduced, in favor of independent continuation by the meditators. Figure 1 gives a schematic overview of the eightsession program spanning 4 weeks. 


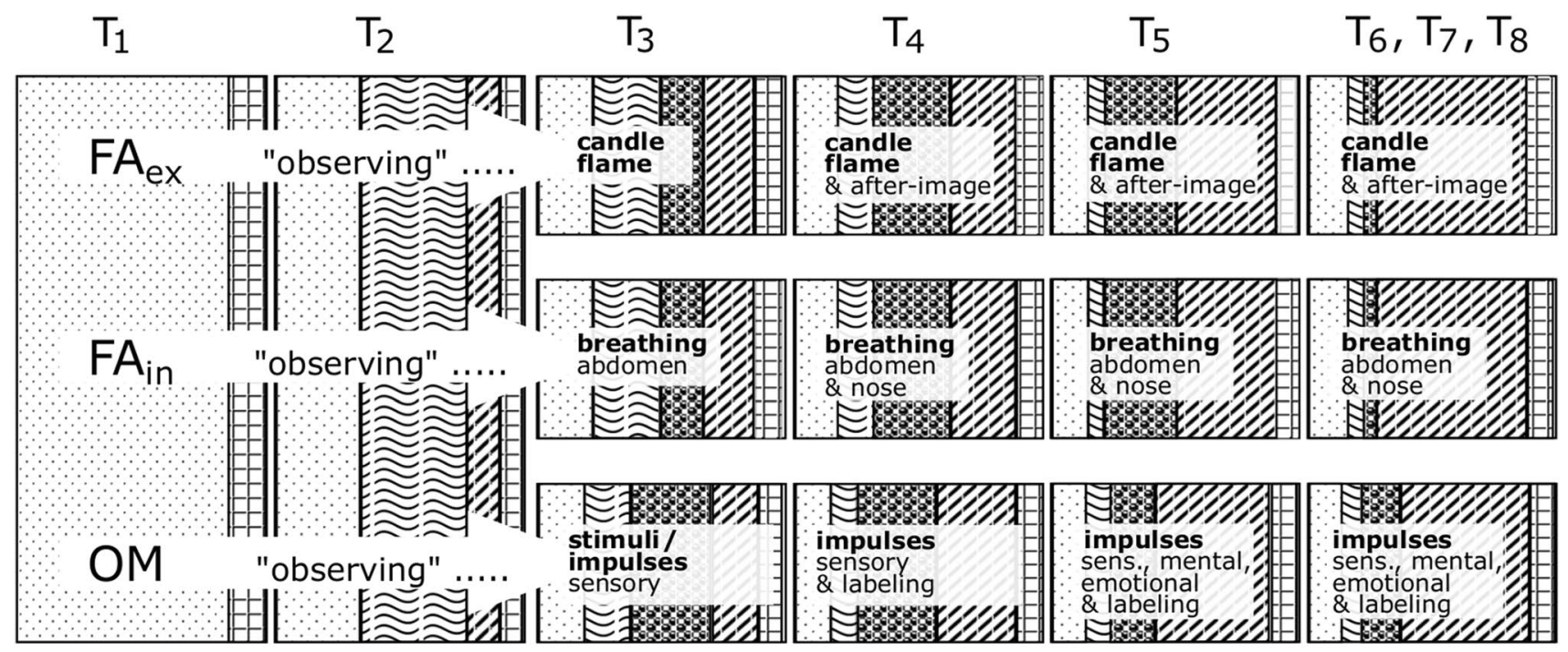

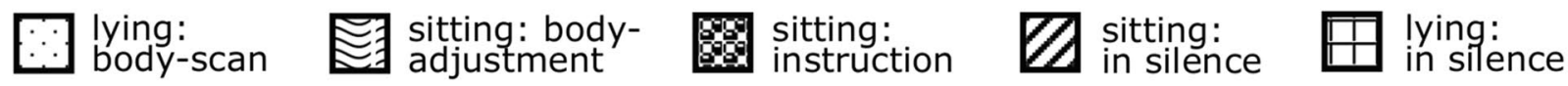

Fig. 1 Schematic sequence of the meditation intervention: three groups $\left(\mathrm{FA}_{\mathrm{ex}}, \mathrm{FA}_{\mathrm{in}}, \mathrm{OM}\right)$, eight group sessions $\left(\mathrm{T}_{1}-\mathrm{T}_{8}\right)$. After the two introductory sessions, every session, for every group, contains five different phases: (1) lying: body scan; (2) sitting: mindful adjustment of one's sitting posture; (3) sitting: instructions for the respec-

\section{Measures}

\section{Measures of Learned Automaticity in the Dot Pattern Expectancy Paradigm}

In the experiment, the dot pattern expectancy paradigm (DPX; Jones et al., 2010) was employed to measure overcoming learned automaticity. This task is a more demanding variation of the $\mathrm{AX}$ continuous performance test (AXCPT; Servan-Schreiber et al., 1996). In the AX-CPT, participants are shown a cue stimulus followed by a probe stimulus. The cue stimulus may be either of the letters A or $\mathrm{B}$, while the probe stimulus may be either of the letters $\mathrm{X}$ or Y. Participants are asked to respond to the probe stimulus: when they have seen an A and then an X, they should press the "target" button on a keyboard; but if they have seen an A followed by a Y, a B followed by an X, or a B followed by a Y, they should press the "non-target" button.

The A-X combination occurs in $70 \%$ of the cases, while $\mathrm{A}-\mathrm{Y}, \mathrm{B}-\mathrm{X}$, and $\mathrm{B}-\mathrm{Y}$ make up $10 \%$ of the trials each. Thus, participants form a habit of pressing the "target" button; it becomes an overlearned routine. A tendency to press the "target" button even if an A was followed by a Y reflects a mechanistic or automatic response: seeing the A-cue can trigger the habit to press the "target" button without considering the non-target $\mathrm{Y}$-probe. Thus, A-Y errors tive meditation technique; (4) sitting: independent continuation of the instructions in silence; (5) lying: independent continuation of the instructions in silence. Different textures symbolize the proportions of different contents of the respective session

highlight the strength of habitual automaticity and, thus, the intensity of the cognitive conflict in overcoming it.

In the DPX-paradigm, each stimulus is an arrangement of three to four dots, comparable to braille letters. The DPX utilizes one specific dot pattern as the target cue (A-cue), one different dot pattern as the target probe (X-probe), and a variety of other different patterns as non-target cues (B-cues) or non-target probes (Y-probes). These non-target cue and probe (B and $\mathrm{Y}$ ) stimuli are picked randomly from an array of five different arrangements each. Like in the AX-CPT, participants are instructed to classify only A-X sequences as "target" and any other sequence (A-Y, B-X, or $\mathrm{B}-\mathrm{Y}$ ) as "non-target." Likewise, to establish a predominant response bias, the DPX consists of $70 \%$ A-X sequences, whereas the other trial types are represented in only $10 \%$ of the trials each. Participants were shown a total of 180 cueprobe sequences. Figure 2 offers a visual representation of the DPX-paradigm.

According to signal detection theory (MacMillan \& Creelman, 1991), sensitivity $d$-prime ( $\left.d^{\prime}\right)$ served as a measure of performance on the task and response conservativity bias ( $c$-bias) as a measure of response strategy. Corrections for extreme values in hit rates or false alarms were applied following the log-linear approach (Snodgrass \& Corwin, 1988). To compute $d$ ', the z-transformed rate of incorrect positive responses to $\mathrm{A}-\mathrm{Y}$ trials (false alarms) was 
Fig. 2 A "target" response is required to an X-probe only when it follows an A-cue by pressing a specific button. Nontarget responses are made after all other types (A-Y, B-X, and $\mathrm{B}-\mathrm{Y}$ ) by pressing a different button. The first two stimuli in the depicted sequence represent a "target" A-X trial; the third and fourth stimuli together represent a "non-target" A-Y trial; "nontarget" B-X and B-Y trials are not depicted here

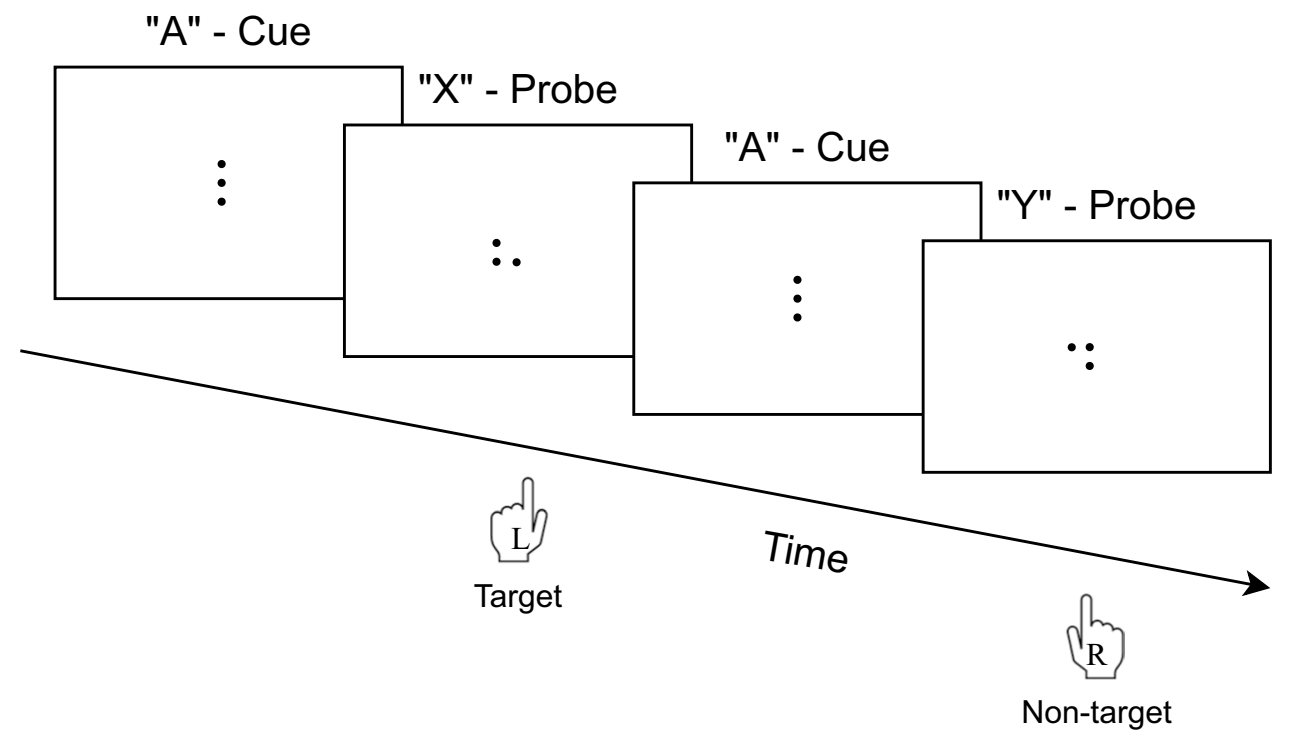

subtracted from the $\mathrm{z}$-transformed rate of correct positive responses to $\mathrm{A}-\mathrm{X}$ trials (hits). The initial valid $\mathrm{A}-\mathrm{cue}$ in non-target $\mathrm{A}-\mathrm{Y}$ trials can trigger the predominant response trained by the overrepresentation of A-X trials. An A-Y false alarm represents a failure to recognize the subsequent invalid $\mathrm{Y}$-probe and, in turn, indicates an erroneous automatic response. Therefore, better results in terms of A-cue $d$ ' represent a better propensity to overcome learned automaticity. The $c$-bias represents the distance between the criterion and the point where each response is chosen with equal frequency (in standard deviation units). Similar to $d$, the $c$-bias was calculated for A-cue sequences by averaging the $\mathrm{z}$-transformed false alarm rate and the $\mathrm{z}$-transformed hit rate, and then multiplying the result by a negative one. As a result, lower values of $c$-bias indicate a bias toward responding negatively ("non-target") rather than positively ("target").

\section{Measures of Learned and Instructed Automaticity in the NEXT-Paradigm}

The NEXT-paradigm was employed as an additional measure of automaticity (Meiran et al., 2015). First, participants received two sets of instructions: one to be implemented when red stimuli are presented on the screen, and a different one to be implemented when the stimuli are green. Then, they were presented with several instances of red stimuli to develop a routine in responding. Suddenly, the stimuli appeared in green color, which required a different type of response. The challenge in responding to green stimuli is twofold: (1) to not execute the routinized reaction and (2) to keep the new set of instructions for green stimuli in mind and respond accordingly.
In total, the NEXT-paradigm consists of 110 blocks of choice tasks. Each block begins with instructions for the current choice task followed by a "NEXT-phase" and then a "GO-phase." First, the participants were instructed that the left key "A" and right key "L" on a computer keyboard correspond to two symbols that appeared on the respective side of the screen. Furthermore, one of those keys was declared the NEXT-key. The subsequent NEXT-phase consisted of 0-5 instances of one of the symbols appearing on the screen in red color. Participants should react by pressing the assigned NEXT-key on a keyboard, regardless of whether the stimulus was initially instructed to the left or right key. Finally, during the GO-phase, 2 instances of a green stimulus appeared on the screen, to which participants should react by pressing the respective key that corresponds to the symbol as instructed initially. Figure 3 offers a visual representation of the NEXT-paradigm.

The instruction at the beginning of each block, which is only applicable during the GO-phase, acts as a source of conflict during the NEXT-phase. Critically, in some NEXTtrials, the NEXT-key corresponds to the presented stimulus (compatible trials); in others, it does not (incompatible trials). This task comprises two rules that are present at the same time and stand in conflict with each other. Depending on the color of the stimuli, the participant has to decide which rule to disregard and which one to implement in its place.

To measure the latency caused by the cognitive conflict that had to be overcome, the reaction time (RT) interference for the NEXT- and the GO-phase were computed separately by calculating the difference in mean RTs between incompatible and compatible trials for each phase. To deal with RT outliers, we applied the median absolute deviation method (see Leys et al., 2013) to RTs separately for each condition 


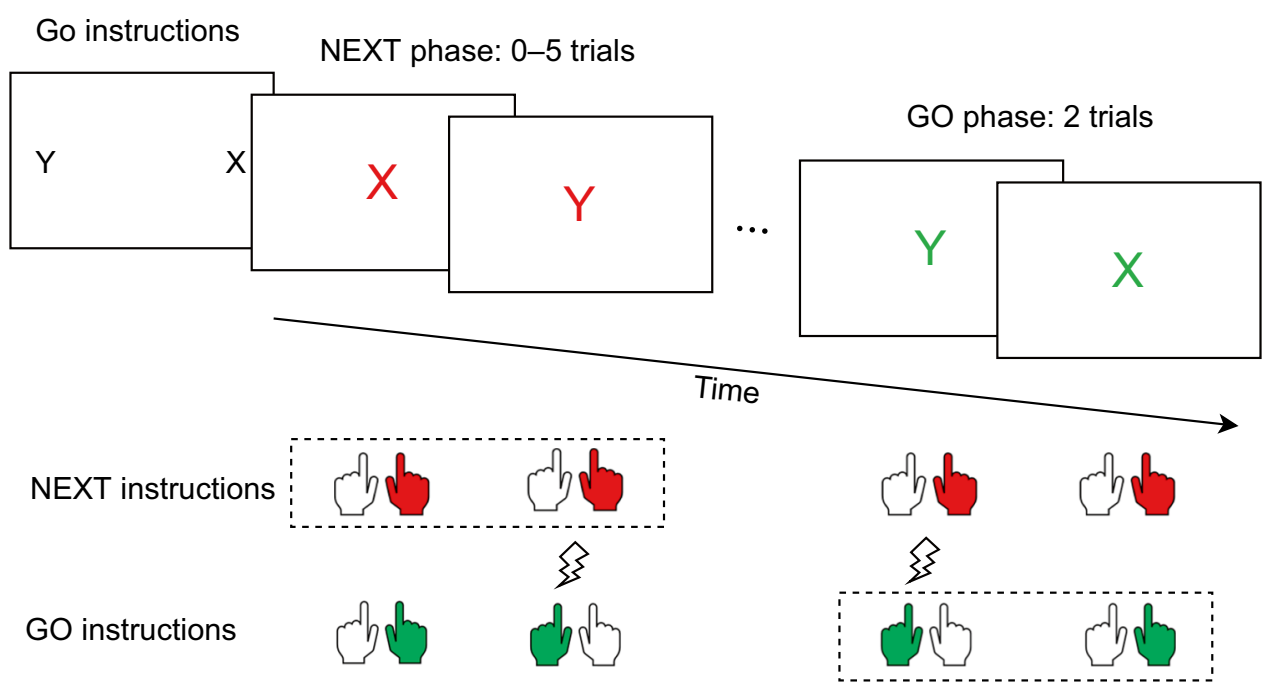

Fig. 3 First, participants received instructions on how to respond during the GO-phase of the upcoming block. Second, during the NEXTphase indicated through red stimuli, they are required to advance the screen using the NEXT-key. When green stimuli appear, participants are required to implement the instructions received at the beginning of the trial instead of pressing the NEXT-key. The correct responses

of each factor and participant. The NEXT-paradigm offers an elegant opportunity to distinguish between learned and instructed automaticity. Firstly, the difference in RTs between incompatible and compatible trials in the NEXTphase describes how a recently instructed response that is only applicable to the GO-phase interferes with the response required for NEXT-phase, the latter of which remains identical during the entire task and, thus, is executed repeatedly. Thus, it reflects a measure of instructed automaticity (Meiran et al., 2015). Secondly, the NEXT-paradigm offers another measure reflecting learned automaticity. Vice versa, the repeatedly executed NEXT-response might become a learned habitual tendency that interferes with the execution of the instructed stimulus-response pattern in the GO-phase. Therefore, the difference in RTs between incompatible and compatible trials in the GO-phase reflects the interference caused by the overlearned NEXT-response and, thus, represents a measure of learned automaticity.

As an additional performance measure, we employed the bin scoring method (see Hughes et al., 2014) by combining error rates with RTs. In incompatible GO-trials, the participant had to give a response that differed from the previous trials. Due to the additional cognitive effort required to swap the dominant rule in incompatible trials, these usually result in longer RTs and a higher error rate. All participants' RT differences between compatible and incompatible trials were collated, ordered into deciles (bins), and assigned a score between 1 (best/fastest 10\% of trials) and 10 (worst/ slowest $10 \%$ of trials); incorrectly answered incompatible trials were assigned a penalty score of 20 regardless of their are framed with a dashed line. The correct response during the NEXT-phase may differ from the initially instructed GO-response; similarly, the correct response during the GO-phase may differ from the habitual NEXT-response. Those situations, indicated with a flash, are incompatible trials, and the others are compatible trials

corresponding RT. Finally, each participant's scores were summed to form a single bin score, representing their performance in resolving the cognitive conflict. Participants did not commit any errors during the NEXT-phase of each trial; hence, there is no bins measure for overcoming instructed automaticity based on the NEXT-phase, but only for the measure of learned automaticity based on the GO-phase.

\section{Results}

\section{Convergence and Divergence in the Measures of Automaticity in the NEXT-Paradigm}

The measures of RT interference for overcoming each type of automaticity in the NEXT-paradigm bore no correlation with each other, $r=0.025, p=0.833$. This finding indicates the distinctness of either type of automaticity and suggests the absence of a trade-off in being proficient in overcoming either one. Furthermore, the measures on overcoming learned automaticity of the DPX validate those of the NEXT-paradigm: Sensitivity in the DPX (A-cue $d$ ') was negatively related to both (negatively scored) measures of the GO-phase of the NEXT-paradigm, namely the RT interference $(r=-0.42, p<0.001)$ and the bins $(r=-0.25$, $p=0.031$ ), whereas $c$-bias was positively related to both the RT interference $(r=0.29, p=0.013)$ and the bins $(r=0.41$, $p<0.001)$. Therefore, the RT interference between incompatible and compatible trials during the GO-phase of the 
Fig. 4 Overview of the results on performance (A) and response bias (B) in the DPX. Error bars represent one standard error of the mean. Effect strengths obtained through planned contrasts are superimposed below the graph. Asterisks indicate significant results: $* p<.05, * * p<.01, * * * p<.001$

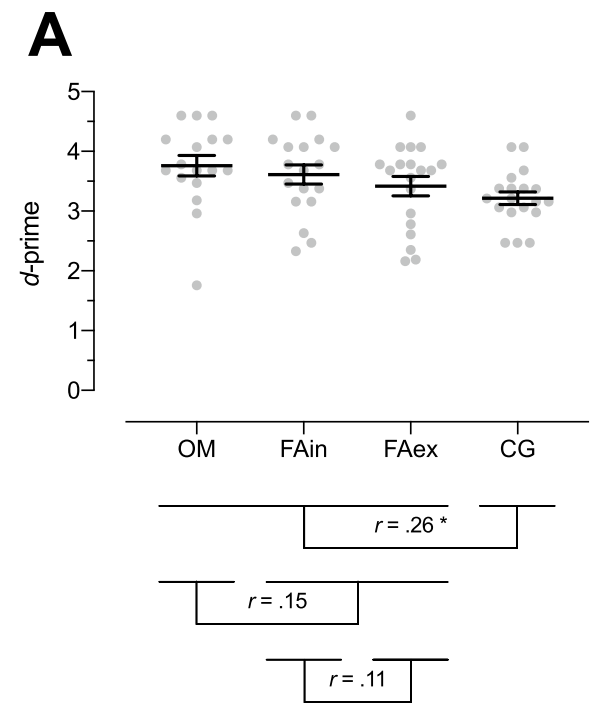

NEXT-paradigm does indeed capture the participants' aptitude at overcoming learned automaticity.

\section{Overcoming Learned Automaticity in the Dot Pattern Expectancy Paradigm}

The participants trained in OM meditation performed best at overcoming learned automaticity in the DPX as measured through d' $(M=3.76, S D=0.70)$, followed by the groups that were trained in $\mathrm{FA}_{\text {in }}(M=3.61, S D=0.68)$, $\mathrm{FA}_{\mathrm{ex}}(M=3.42, S D=0.70)$, and the control group $(M=3.22$, $S D=0.45$ ). A univariate analysis of variance (ANOVA) revealed that the effect of the variation in training on sensitivity reached almost significant levels, $F(3,69)=2.43$, $p=0.073, \eta_{\mathrm{p}}^{2}=0.096$. Planned contrasts indicated a significant small difference in performance between the meditation groups and the control group, $t(69)=2.21, p=0.031$, $r=0.26$. Looking at the differences between the meditation groups, there were no significant differences neither between the OM and FA groups $(t(69)=1.29, p=0.201, r=0.15)$ nor between the $\mathrm{FA}_{\text {in }}$ and the $\mathrm{FA}_{\text {ex }}$ group $(t(69)=0.93$, $p=0.356, r=0.11)$. Furthermore, the group trained in OM meditation was most likely to respond negatively to trials as measured through c-bias $(M=0.43, S D=0.20)$, followed by the groups that were trained in $\mathrm{FA}_{\text {in }}(M=0.56, S D=0.21)$, $\mathrm{FA}_{\mathrm{ex}}(M=0.63, S D=0.20)$, and the control group $(M=0.77$, $S D=0.21)$. There was a significant large effect of the variation in training on c-bias $(F(3,69)=8.92, p<0.001$, $\eta_{\mathrm{p}}{ }^{2}=0.280$ ). Further investigation using planned contrasts revealed a significant medium-sized difference between the meditation groups and the control group $(t(69)=4.33$, $p<0.001, r=0.46)$. Comparing the meditation groups, there was a medium-size effect indicating that the participants in the group trained in OM meditation were significantly more likely to respond negatively to trials than those in the FA condition $(t(69)=2.75, p=0.008, r=0.31)$. The differences between $\mathrm{FA}_{\mathrm{in}}$ and $\mathrm{FA}_{\mathrm{ex}}$ were not significant $(t(69)=1.00$, $p=0.321, r=0.12$ ).

These results on overcoming learned automaticity in the DPX-paradigm as visualized in Fig. 4 indicate that the training in meditation may have made the subjects more alert toward questioning the present stimulus instead of blindly responding positively due to the overrepresentation of positive trials. Meditators performed better in overcoming learned automaticity than the control group, lending support to our first hypothesis. Furthermore, while OM meditation did not increase their performance to a greater extent than FA meditation, it made them maintain a more questioning attitude toward their automated behavioral patterns, resulting in a higher rate of negative responses.

\section{Overcoming Learned Automaticity in the NEXT-Paradigm}

The participants trained in OM meditation had the lowest RT interference during the GO-phase $(M=-11.1, S D=15.0)$. Surprisingly, they responded faster to incompatible trials than they did to compatible ones. The OM group was followed by the group trained in $\mathrm{FA}_{\text {in }}(M=8.9, S D=22.8)$, the control group $(M=12.7, S D=15.9)$, and the group trained in $\mathrm{FA}_{\mathrm{ex}}(M=18.2, S D=25.8)$. A univariate ANOVA revealed a significant large main effect of meditation group allocation on RT interference $(F(3,69)=6.80, p<0.001$, and $\left.\eta_{\mathrm{p}}{ }^{2}=0.228\right)$. Planned contrasts indicated no significant difference between the meditation groups and the control group $(t(69)=1.56, p=0.126, r=0.24)$. Interestingly, a post hoc test using Fisher's LSD revealed that the control group differs significantly and largely from the OM group $(p=0.001$, $d=1.530$ ). Differentiating between the meditation groups, the group trained in OM performed significantly and largely 
Fig. 5 Overview of the results on overcoming learned automaticity in the NEXT-paradigm assessed through reaction time interference (in [ms]) (A) and bins (B). Error bars represent one standard error of the mean. Effect strengths obtained through planned contrasts are superimposed below the graph. Asterisks indicate significant results: $* p<.05, * * p<.01$, $* * * p<.001$

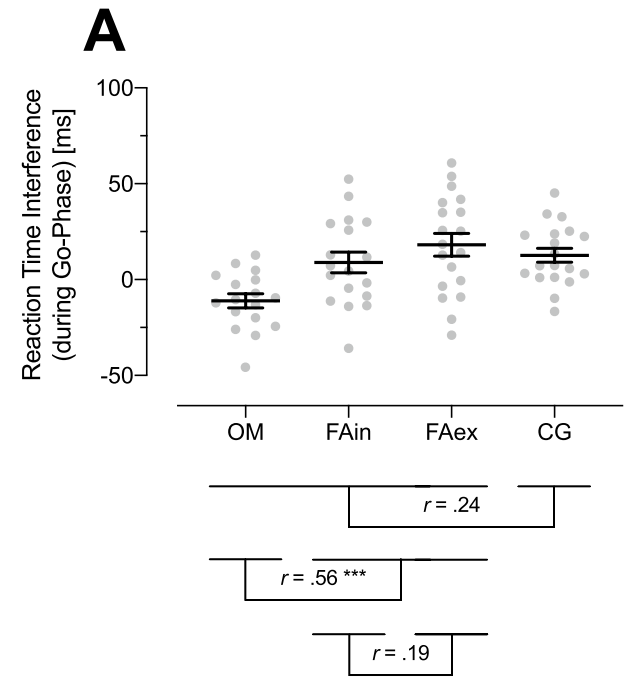

better than the FA groups $(t(69)=4.55, p<0.001, r=0.56)$. The differences between $\mathrm{FA}_{\text {in }}$ and $\mathrm{FA}_{\mathrm{ex}}$ were not significant $(t(34.840)=1.16, p=0.256, r=0.19)$.

Furthermore, adding error rates to the equation to compute bin scores, the participants trained in OM meditation performed best $(M=679, S D=157)$, followed by the groups that were trained in $\mathrm{FA}_{\text {in }}(M=761, S D=181)$, then $\mathrm{FA}_{\mathrm{ex}}(M=881, S D=194)$, and the control group $(M=929$, $S D=183)$. There was a significant large effect of the variation in training on the bin score $(F(3,69)=7.19, p<0.001$, $\left.\eta_{\mathrm{p}}{ }^{2}=0.238\right)$. Employing planned contrasts, there was a significant medium-sized difference between the meditation groups and the control group $(t(69)=3.24, p=0.002$, $r=0.36$ ). Between the meditation groups, the OM group and the FA groups differed largely from each other $(t(69)=2.70$, $p=0.009, r=0.31)$. Furthermore, there was a medium-sized difference between the $\mathrm{FA}_{\mathrm{in}}$ and the $\mathrm{FA}_{\mathrm{ex}}$ group $(t(69)=2.03$, $p=0.046, r=0.24$ ).

Taken together, the findings on overcoming learned automaticity in the NEXT-paradigm as visualized in Fig. 5 further substantiate our first hypothesis: meditation, regardless of technique, has benefited participants' ability to overcome learned automaticity. Furthermore, OM practice was particularly beneficial compared to FA practice in this task only, lending indicative support to our second hypothesis.

\section{Overcoming Instructed Automaticity in the NEXT-Paradigm}

The group trained in $\mathrm{FA}_{\mathrm{ex}}$ showed the greatest performance in overcoming instructed automaticity as represented by the RT interference during the NEXT-phase of the eponymous task $(M=2.8, S D=12.2)$, followed by the control group $(M=4.2, S D=12.5)$, the group trained in OM $(M=7.3, S D=9.0)$, and, lastly, the group trained in FAin $(M=10.4, S D=18.1)$. A univariate ANOVA revealed no significant effect of meditation style on RT interference $\left(F(3,69)=1.16, p=0.330, \eta_{\mathrm{p}}{ }^{2}=0.048\right)$. Further explorative computation of planned contrasts also yielded no significant results when comparing the meditation groups versus the control group $(t(69)=0.73, p=0.465, r=0.09$ ). Furthermore, there were no significant differences between the meditation groups, neither comparing the OM group to the two FA groups $(t(69)=0.18, p=0.855, r=0.02)$ nor comparing the FAin group to the FAex group $(t(69)=1.71$, $p=0.091, r=0.20$ ). Explorative post hoc pairwise comparisons using Fisher's LSD did not reach significant levels either $(p s=0.091-0.502)$.

The findings on overcoming instructed automaticity in the NEXT-paradigm as visualized in Fig. 6 are contrary to our first hypothesis: there was no impact of meditation, regardless of technique, on overcoming instructed automaticity. They further highlight the notion of automaticity as a multifaceted construct (Stahl et al., 2014).

\section{Discussion}

Situations solved by automatic responses are widespread, representing a ready example of the Janus-faced nature of automaticity: it allows individuals to operate efficiently amidst consistent demands, but also bears the potential for errors if demands suddenly change. Consequently, empowering individuals to evaluate the appropriateness of their automatisms in light of each situation's peculiar demands and to decide on the most suitable action can serve to prevent adversity. We hypothesized, first, that meditation training, regardless of technique, improves overcoming automaticity; and, second, that training in OM does so to a greater extent than training in FA.

The intervention improved participants' performance in overcoming learned automaticity across two tasks, 


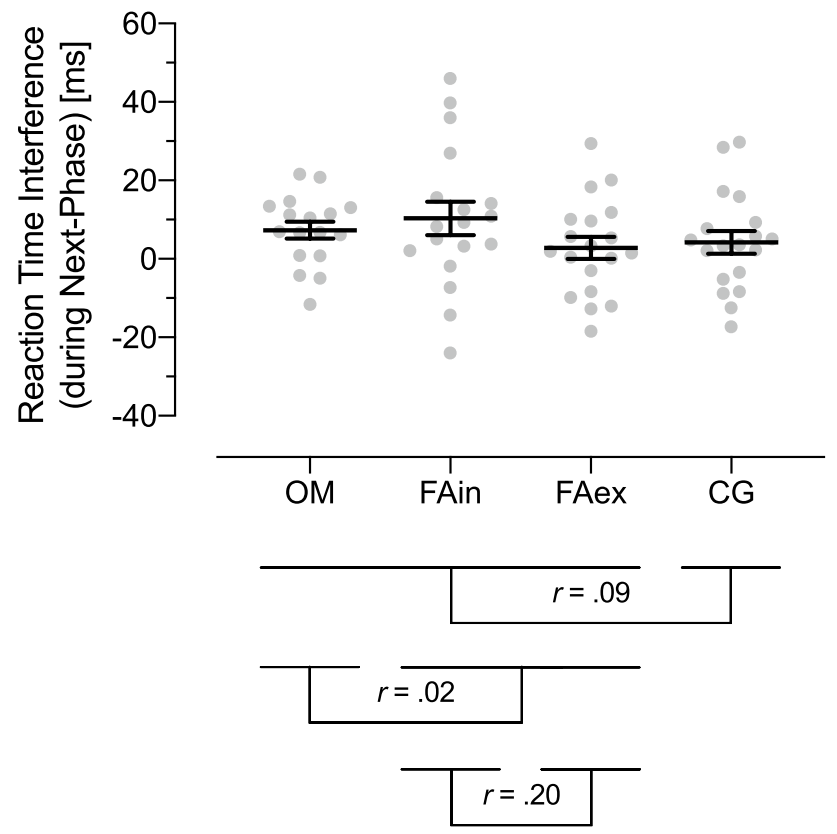

Fig. 6 Overview of the results on overcoming instructed automaticity in the NEXT-paradigm assessed through reaction time interference (in [ms]). Error bars represent one standard error of the mean. Effect strengths obtained through planned contrasts are superimposed below the graph, though none are statistically significant

supporting our first hypothesis. However, their performance in overcoming instructed automaticity remained unaffected. Further, meditation training has made participants respond more conservatively in one task: They inhibited their overlearned response more often than non-meditators, indicating that they took a more questioning stance toward the appropriateness of their habit. This finding held true even for situations where the automated response would have been correct, and it is more pronounced for the group trained in OM rather than FA. Finally, comparing the groups regarding their performance in overcoming learned automaticity yielded ambiguous results: while the groups did not differ in one of two tasks, OM practitioners performed better than FA practitioners in the other one.

Meditation training has supported the participants' ability to overcome erroneous automatic responses in routinized situations that have been executed frequently, but not in novel ones where the adequate action has merely been explained instead of practiced. In this regard, OM meditation may be a more promising practice than FA, but further research is needed to substantiate this notion.
Effects of Meditation on Overcoming Different Types of Automaticity

During meditation, regardless of technique, practitioners continually recognize, inhibit, and let go of distractions that interfere with their practice (Lutz et al., 2008). This suggests that meditation practice may be universally suitable for preventing arising action tendencies from becoming dominant over alternative responses. However, the distractions that arise during meditation stem from information stored in long-term memory (Andrews-Hanna et al., 2018; Ellamil et al., 2016), rather than from earlier instructions that are actively represented in working memory (Cole et al., 2017). Analogously, in learned automaticity, the overlearned automated response is stored in long-term memory, whereas instructed automaticity relies on the instructions being held in working memory instead. Furthermore, automaticity is a multifaceted construct comprised of, among others, the inhibition of an ongoing response (reactive stopping; e.g., Cai et al., 2011), and preparations to selectively stop an arising response tendency (response selection, or selective proactive stopping; Aron, 2011; Stahl et al., 2014). The ability to stop an ongoing response can support overcoming learned automaticity, while the ability to stop an arising response tendency can support overcoming instructed automaticity. Notably, meditation practice has been found to improve behavioral inhibition but not response selection when different response options compete (Greenberg et al., 2013). Taken together, practice in meditation may resemble situations of learned automaticity more closely than it does situations of instructed automaticity. The distractions encountered in meditation possess properties comparable to overlearned action tendencies, and both can be disengaged from using similar cognitive operations. In turn, meditation training can reduce one's likelihood of falling victim to erroneous habitual responding stemming from repeated execution.

\section{Differences Between Meditation Techniques}

The divergence in outcomes between techniques may be due to different mental operations that are trained in either technique (e.g., Lutz et al., 2008). Although the requirement to avoid engaging in distracting thoughts is common for both techniques, they differ in their approach. More specifically, FA practitioners train to stop every distraction as soon as they recognize it and refocus their attention on their intended object. By contrast, OM practitioners do not focus on any object, but rather observe the upcoming impulse and their response to it. When a thought emerges, they restrain their natural tendency to expand on it (Desbordes et al., 2015). Therefore, OM practitioners train to not engage with unwanted impulses and to not let them expand to dominate 
other sensations; instead, they clearly aim to notice the content and quality of the impulse from an observer's perspective without attachment. Thus, when OM practitioners are confronted with a cognitive conflict, their improved monitoring ability should make them less prone to allow a repeatedly executed response to dominate other options and to select the appropriate response in its place.

\section{Limitations and Future Research}

The present study provides compelling findings, but it is not without limitations. First, while the experimental design at hand displays high internal validity, it is unclear how well the results translate to real-world situations in which a person has to make a decision in the face of automaticity. Second, positive beliefs about training may affect its outcomes (Tsai et al., 2018). Recruiting an active control group that performs a sham training instead of the wait list control group may be a possible mitigation strategy. Third, the dose of this intervention was similar to established literature on discerning the effects of meditation compared to a control group and sufficient in doing so. However, the results on distinguishable effects of different meditation techniques are indicative but not yet conclusive. Since both OM and FA meditations overlap partially in their utilization of cognitive mechanisms with the cognitive differences between the techniques being subtle and nuanced, their outcomes may only diverge after a longer period of training. Thus, future research may utilize a larger dose to elucidate this promising avenue. Fourth, this study considered neither reason for a dropout nor participants' motivation and their expectations (e.g., relaxation, cognitive enhancement, knowledge of traditional literature, ethical aspirations). For example, Jensen et al. (2012) demonstrated a significant, motivation-related influence on the task performance of meditators. Future work should take into account both the reasons for quitting training ahead of schedule and the basic motivation for meditation. This would allow testing for specific relations between both. Lastly, the conceptualization of OM meditation as non-judgemental awareness of present-moment experiences does not incorporate an ethical dimension, which is important in the traditional technique (Ditrich, 2017; Monteiro et al., 2015; Nilsson \& Kazemi, 2016). While this "narrow" conceptualization-found in most existing research - is derived from Buddhist mindfulness, it should not be mistaken to represent it in its entirety.

Author Contribution TM designed the study and analyzed the data. MW designed the study, narrated the guided meditation sessions, executed the intervention, and wrote the paper. SM analyzed the data and wrote the paper. MF provided supervisory guidance. EJ analyzed the data and wrote the paper. SH executed the intervention. GH contributed to the data collection. All authors approved the final version of the manuscript for submission.

Funding Open access funding provided by University of Innsbruck and Medical University of Innsbruck.

\section{Declarations}

Ethics Approval The present study was approved by the Board for Ethical Issues of the University of Innsbruck in accordance with the Declaration of Helsinki. All participants gave their informed consent prior to their inclusion in the study. Details that might disclose their identity have been omitted.

Conflict of Interest The authors declare no competing interests.

Open Access This article is licensed under a Creative Commons Attribution 4.0 International License, which permits use, sharing, adaptation, distribution and reproduction in any medium or format, as long as you give appropriate credit to the original author(s) and the source, provide a link to the Creative Commons licence, and indicate if changes were made. The images or other third party material in this article are included in the article's Creative Commons licence, unless indicated otherwise in a credit line to the material. If material is not included in the article's Creative Commons licence and your intended use is not permitted by statutory regulation or exceeds the permitted use, you will need to obtain permission directly from the copyright holder. To view a copy of this licence, visit http://creativecommons.org/licenses/by/4.0/.

\section{References}

Ainsworth, B., Eddershaw, R., Meron, D., Baldwin, D. S., \& Garner, M. (2013). The effect of focused attention and open monitoring meditation on attention network function in healthy volunteers. Psychiatry Research, 210(3), 1226-1231. https://doi.org/10. 1016/j.psychres.2013.09.002

Anālayo. (2003). Satipatthāna-The Direct Path to Realization. Windhorse Publications.

Andrews-Hanna, J. R., Irving, Z. C., Fox, K. C. R., Spreng, R. N., \& Christoff, K. (2018). The neuroscience of spontaneous thought. In K. Christoff \& K. C. R. Fox (Eds.), The Oxford handbook of spontaneous thought: Mind-wandering, creativity, and dreaming. Oxford University Press.

Aron, A. R. (2011). From reactive to proactive and selective control: Developing a richer model for stopping inappropriate responses. Biological Psychiatry, 69(12), e55-68. https://doi.org/10.1016/j. biopsych.2010.07.024

Bishop, S. R., Lau, M., Shapiro, S. L., Carlson, L. E., Anderson, N. D., Carmody, J., Segal, Z. V., Abbey, S., Speca, M., Velting, D., \& Devins, G. M. (2004). Mindfulness: A proposed operational definition. Clinical Psychology: Science and Practice, 11(3), 230-241. https://doi.org/10.1093/clipsy.bph077

Cahn, B. R., \& Polich, J. (2009). Meditation (Vipassana) and the P3a event-related brain potential. International Journal of Psychophysiology, 72(1), 51-60. https://doi.org/10.1016/j.ijpsycho.2008. 03.013

Cai, W., Oldenkamp, C. L., \& Aron, A. R. (2011). A proactive mechanism for selective suppression of response tendencies. The Journal of Neuroscience, 31(16), 5965-5969. https://doi.org/10.1523/ jneurosci.6292-10.2011 
Chiesa, A., Calati, R., \& Serretti, A. (2011). Does mindfulness training improve cognitive abilities? A systematic review of neuropsychological findings. Clinical Psychology Review, 31(3), 449-464. https://doi.org/10.1016/j.cpr.2010.11.003

Christoff, K., Irving, Z. C., Fox, K. C. R., Spreng, R. N., \& AndrewsHanna, J. R. (2016). Mind-wandering as spontaneous thought: A dynamic framework. Nature Reviews Neuroscience, 17(11), 718-731. https://doi.org/10.1038/nrn.2016.113

Cole, M. W., Braver, T. S., \& Meiran, N. (2017). The task novelty paradox: Flexible control of inflexible neural pathways during rapid instructed task learning. Neuroscience and Biobehavioral Reviews, 81(A), 4-15. https://doi.org/10.1016/j.neubiorev.2017. 02.009

Colzato, L. S., Szapora, A., \& Hommel, B. (2012). Meditate to create: The impact of Focused-Attention and Open-Monitoring training on convergent and divergent thinking. Frontiers in Psychology, 3, 116. https://doi.org/10.3389/fpsyg.2012.00116

Creswell, J. D., Way, B. M., Eisenberger, N. I., \& Lieberman, M. D. (2007). Neural correlates of dispositional mindfulness during affect labeling. Psychosomatic Medicine, 69(6), 560-565. https://doi.org/10.1097/PSY.0b013e3180f6171f

Desbordes, G., Gard, T., Hoge, E. A., Hölzel, B. K., Kerr, C., Lazar, S. W., Olendzki, A., \& Vago, D. R. (2015). Moving beyond mindfulness: Defining equanimity as an outcome measure in meditation and contemplative research. Mindfulness, 6(2), 356372. https://doi.org/10.1007/s12671-013-0269-8

Ditrich, T. (2017). The conceptualization and practice of mindfulness: Buddhist and secular perspectives. In T. Ditrich, R. Wiles, \& B. Lovegrove (Eds.), Mindfulness and education: Research and practice (pp. 3-32). Cambridge Scholars Publishing.

Eagle, D. M., Bari, A., \& Robbins, T. W. (2008). The neuropsychopharmacology of action inhibition: Cross-species translation of the stop-signal and go/no-go tasks. Psychopharmacology (berl), 199(3), 439-456. https://doi.org/10.1007/s00213-008-1127-6

Ellamil, M., Fox, K. C. R., Dixon, M. L., Pritchard, S., Todd, R. M., Thompson, E., \& Christoff, K. (2016). Dynamics of neural recruitment surrounding the spontaneous arising of thoughts in experienced mindfulness practitioners. NeuroImage, 136, 186-196. https://doi.org/10.1016/j.neuroimage.2016.04.034

Fox, K. C. R., Dixon, M. L., Nijeboer, S., Girn, M., Floman, J. L., Lifshitz, M., Ellamil, M., Sedlmeier, P., \& Christoff, K. (2016). Functional neuroanatomy of meditation: A review and metaanalysis of 78 functional neuroimaging investigations. Neuroscience \& Biobehavioral Reviews, 65, 208-228. https://doi.org/10. 1016/j.neubiorev.2016.03.021

Gallant, S. N. (2016). Mindfulness meditation practice and executive functioning: Breaking down the benefit. Consciousness and Cognition, 40, 116-130. https://doi.org/10.1016/j.concog.2016. 01.005

Gethin, R. (2011). On some definitions of mindfulness. Contemporary Buddhism, 12(1), 263-279. https://doi.org/10.1080/14639 947.2011.564843

Grabovac, A. D., Lau, M. A., \& Willett, B. R. (2011). Mechanisms of mindfulness: A Buddhist psychological model. Mindfulness, 2(3), 154-166. https://doi.org/10.1007/s12671-011-0054-5

Greenberg, J., Reiner, K., \& Meiran, N. (2013). "Off with the old": Mindfulness practice improves backward inhibition. Frontiers in Psychology, 3, 618. https://doi.org/10.3389/fpsyg.2012.00618

Hart, W. (1987). The art of living: Vipassana meditation as taught by S. N. Goenka. HarperCollins

Holas, P., \& Jankowski, T. (2013). A cognitive perspective on mindfulness. International Journal of Psychology, 48(3), 232-243. https://doi.org/10.1080/00207594.2012.658056

Hommel, B., \& Colzato, L. S. (2017). Meditation and metacontrol. Journal of Cognitive Enhancement, 1(2), 115-121. https://doi. org/10.1007/s41465-017-0017-4
Hughes, M. M., Linck, J. A., Bowles, A. R., Koeth, J. T., \& Bunting, M. F. (2014). Alternatives to switch-cost scoring in the task-switching paradigm: Their reliability and increased validity. Behavior Research Methods, 46(3), 702-721. https://doi.org/10. 3758/s13428-013-0411-5

Inzlicht, M., Bartholow, B. D., \& Hirsh, J. B. (2015). Emotional foundations of cognitive control. Trends in Cognitive Sciences, 19(3), 126-132. https://doi.org/10.1016/j.tics.2015.01.004

Isbel, B., \& Mahar, D. (2015). Cognitive mechanisms of mindfulness: A test of current models. Consciousness and Cognition, 38, 50-59. https://doi.org/10.1016/j.concog.2015.10.005

Jensen, C. G., Vangkilde, S., Frokjaer, V., \& Hasselbalch, S. G. (2012). Mindfulness training affects attention-Or is it attentional effort? Journal of Experimental Psychology: General, 141(1), 106-123. https://doi.org/10.1037/a0024931

Jones, J. A. H., Sponheim, S. R., \& MacDonald, A. W. (2010). The dot pattern expectancy task: Reliability and replication of deficits in schizophrenia. Psychological Assessment, 22(1), 131-141. https:// doi.org/10.1037/a0017828

Kang, Y., Gruber, J., \& Gray, J. R. (2013). Mindfulness and de-automatization. Emotion Review, 5(2), 192-201. https://doi.org/10.1177/ 1754073912451629

Kurzban, R. (2016). The sense of effort. Current Opinion in Psychology, 7, 67-70. https://doi.org/10.1016/j.copsyc.2015.08.003

Leys, C., Ley, C., Klein, O., Bernard, P., \& Licata, L. (2013). Detecting outliers: Do not use standard deviation around the mean, use absolute deviation around the median. Journal of Experimental Social Psychology, 49(4), 764-766. https://doi.org/10.1016/j.jesp. 2013.03.013

Logan, G. D. (1978). Attention in character-classification tasks: Evidence for the automaticity of component stages. Journal of Experimental Psychology: General, 107(1), 32-63. https://doi.org/10. 1037/0096-3445.107.1.32

Lutz, A., Jha, A. P., Dunne, J. D., \& Saron, C. D. (2015). Investigating the phenomenological matrix of mindfulness-related practices from a neurocognitive perspective. American Psychologist, 70(7), 632-658. https://doi.org/10.1037/a0039585

Lutz, A., Slagter, H. A., Dunne, J. D., \& Davidson, R. J. (2008). Attention regulation and monitoring in meditation. Trends in Cognitive Sciences, 12(4), 163-169. https://doi.org/10.1016/j.tics.2008.01. 005

MacMillan, N. A., \& Creelman, C. D. (1991). Detection theory: A user's guide. Cambridge University Press

Meiran, N., Cole, M. W., \& Braver, T. S. (2012). When planning results in loss of control: Intention-based reflexivity and working-memory. Frontiers in Human Neuroscience, 6, 104. https://doi.org/10. 3389/fnhum.2012.00104

Meiran, N., Liefooghe, B., \& De Houwer, J. (2017). Powerful instructions: Automaticity without practice. Current Directions in Psychological Science, 26(6), 509-514. https://doi.org/10.1177/ 0963721417711638

Meiran, N., Pereg, M., Kessler, Y., Cole, M. W., \& Braver, T. S. (2015). The power of instructions: Proactive configuration of stimulusresponse translation. Journal of Experimental Psychology: Learning, Memory, and Cognition, 41(3), 768-786. https://doi.org/10. 1037/xlm0000063

Melby-Lervåg, M., Redick, T. S., \& Hulme, C. (2016). Working memory training does not improve performance on measures of intelligence or other measures of "far transfer": Evidence from a meta-analytic review. Perspectives on Psychological Science, 11(4), 512-534. https://doi.org/10.1177/1745691616635612

Monteiro, L. M., Musten, R. F., \& Compson, J. (2015). Traditional and contemporary mindfulness: Finding the middle path in the tangle of concerns. Mindfulness, 6(1), 1-13. https://doi.org/10. 1007/s12671-014-0301-7 
Moors, A. (2016). Automaticity: Componential, causal, and mechanistic explanations. Annual Review of Psychology, 67(1), 263-287. https://doi.org/10.1146/annurev-psych-122414-033550

Morrison, A. B., Goolsarran, M., Rogers, S. L., \& Jha, A. P. (2014). Taming a wandering attention: Short-form mindfulness training in student cohorts. Frontiers in Human Neuroscience, 7, 897. https:// doi.org/10.3389/fnhum.2013.00897

Nilsson, H., \& Kazemi, A. (2016). Reconciling and thematizing definitions of mindfulness: The big five of mindfulness. Review of General Psychology, 20(2), 183-193. https://doi.org/10.1037/ gpr0000074

Nyanaponika, T. (1962). The heart of Buddhist meditation. Rider \& Co.

Olendzki, A. (2011). The construction of mindfulness. Contemporary Buddhism, 12(1), 55-70. https://doi.org/10.1080/14639947.2011. 564817

Packard, M. G., \& Goodman, J. (2013). Factors that influence the relative use of multiple memory systems. Hippocampus, 23(11), 1044-1052. https://doi.org/10.1002/hipo.22178

Sala, G., \& Gobet, F. (2019). Cognitive training does not enhance general cognition. Trends in Cognitive Sciences, 23(1), 9-20. https:// doi.org/10.1016/j.tics.2018.10.004

Sebastian, A., Pohl, M. F., Klöppel, S., Feige, B., Lange, T., Stahl, C., Voss, A., Klauer, K. C., Lieb, K., \& Tüscher, O. (2013). Disentangling common and specific neural subprocesses of response inhibition. NeuroImage, 64, 601-615. https://doi.org/10.1016/j. neuroimage.2012.09.020

Servan-Schreiber, D., Cohen, J. D., \& Steingard, S. (1996). Schizophrenic deficits in the processing of context: A test of a theoretical model. Archives of General Psychiatry, 53(12), 1105. https://doi. org/10.1001/archpsyc.1996.01830120037008

Shapiro, S. L., Carlson, L. E., Astin, J. A., \& Freedman, B. (2006). Mechanisms of mindfulness. Journal of Clinical Psychology, 62(3), 373-386. https://doi.org/10.1002/jclp.20237
Snodgrass, J. G., \& Corwin, J. (1988). Pragmatics of measuring recognition memory: Applications to dementia and amnesia. Journal of Experimental Psychology: General, 117(1), 34-50. https://doi. org/10.1037/0096-3445.117.1.34

Stahl, C., Voss, A., Schmitz, F., Nuszbaum, M., Tüscher, O., Lieb, K., \& Klauer, K. C. (2014). Behavioral components of impulsivity. Journal of Experimental Psychology: General, 143(2), 850-886. https://doi.org/10.1037/a0033981

Sumantry, D., \& Stewart, K. E. (2021). Meditation, mindfulness, and attention: A meta-analysis. Mindfulness, 12(6), 1332-1349.

Tang, Y.-Y., Wang, J., Fan, Y., Feng, S., Lu, Q., Yu, Q., Sui, D., Rothbart, M. K., Fan, M., \& Posner, M. I. (2007). Short-term meditation training improves attention and self-regulation. Proceedings of the National Academy of Sciences, 104(43), 17152-17156. https://doi.org/10.1073/pnas.0707678104

Tsai, N., Buschkuehl, M., Kamarsu, S., Shah, P., Jonides, J., \& Jaeggi, S. M. (2018). (Un)great expectations: The role of placebo effects in cognitive training. Journal of Applied Research in Memory and Cognition, 7(4), 564-573. https://doi.org/10.1016/j.jarmac. 2018.06.001

Publisher's Note Springer Nature remains neutral with regard to jurisdictional claims in published maps and institutional affiliations.

Supplementary Information All collected data and the audio files used in the intervention (in German language) are available at the Open Science Framework website (https://osf.io/dcn2f/). 